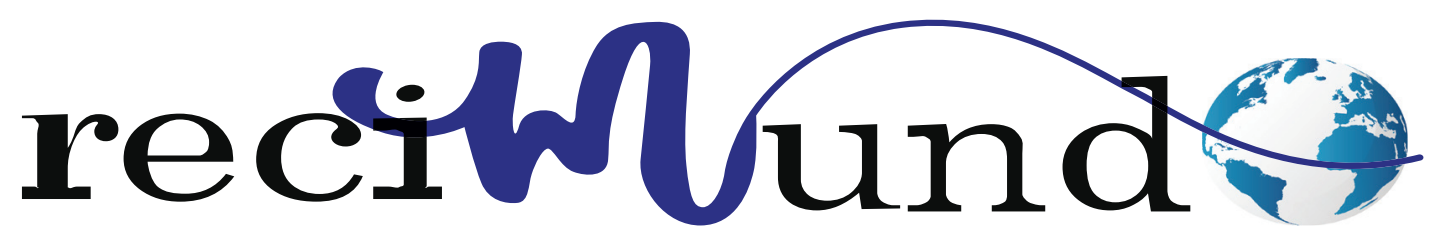

Revista Científica Mundo de la Investigación y el Conocimiento

DOI: 10.26820/recimundo/4.(1).enero.2020.142-151

URL: http://recimundo.com/index.php/es/article/view/736

EDITORIAL: Saberes del Conocimiento

REVISTA: RECIMUNDO

ISSN: 2588-073X

TIPO DE INVESTIGACIÓN: Artículo de Revisión

CÓDIGO UNESCO: 3205 Medicina Interna

PAGINAS: 142-151

\title{
Factores de riesgo y complicaciones del traumatismo craneoencefálico en adulto joven
}

\author{
Risk factors and complications of head trauma in young \\ adults
}

\section{Fatores de risco e complicações de traumatismo craniano em adultos jovens}

Karla Alejandra Paredes Zambrano'; María Soledad Cedeño Veintimilla; Pedro Gerardo De Los Ríos Tomalá3; Francisco Arturo Vaca Morla ${ }^{4}$

RECIBIDO: 18/09/2019 ACEPTADO: 29/10/2019 PUBLICADO: 31/01/2020

1. Médico; Investigador Independiente; Guayaquil, Ecuador; gaby1064@hotmail.com; iD https://orcid.org/00000002-4948-2579

2. Médico; Investigador Independiente; Guayaquil, Ecuador; alejandra_c_11@hotmail.com; iD https://orcid. org/0000-0002-1595-9527

3. Médico Cirujano; Investigador Independiente; Guayaquil, Ecuador, Ecuador; pajr1991@hotmail.com; (D) https://orcid.org/0000-0002-8279-1357

4. Médico Cirujano; Investigador Independiente; Guayaquil, Ecuador; bryanramirez_mh@hotmail.com; (D https:// orcid.org/0000-0002-8324-4072

CORRESPONDENCIA

María Gabriela Castillo Benavides

gaby1064@hotmail.com

Guayaquil, Ecuador 


\section{RESUMEN}

Introducción: El trauma craneoencefálico es un problema de mayor relevancia a nivel mundial que fecta mayormente a la población masculina adulto joven, nuestro estudio esta orientado al análisis de los factores de riesgo y complicaciones del traumatismo craneoencefálico, en nuestro medio la incidencia por el tipo de lesiones del traumatismo craneoencefálico es considerable, que puede elevar el indice de morbilidad y mortalidad si no se diagnostica y trata a tiempo. Metodologia: Este es un estudio transversal, retrospectivo observacional donde se estudia los factores de riesgo y complicaciones del trauma craneoencefálico en adulto joven. Se estudió un total de 100 pacientes que presentaron Tráuma craneoencefálico en adulto joven, estudio realizado en el Hospital Especializado Abel Gilbert Pontón del periodo de Enero a Diciembre del 2015. Resultados: El control adecuado de los factores de riesgo disminuira el indice de traumas craneoencefalico en adulto joven y sus complicaciones.

Palabras clave: Trauma, Craneoencefálico, Factores de Riesgo, Complicaciones, Adulto, Joven.

\section{ABSTRACT}

Introduction: Traumatic brain injury is a problem most relevant worldwide that affect mostly to the adult male population young, our study is oriented to the analysis of risk factors and complications of TCE, in our incidence by type of injury TEC is considerable, which can raise the rate of morbidity and mortality if not diagnosed and treated early. Methodology: This is an observational cross-sectional study, retrospective where risk factors and complications of head trauma in adult is studied. A total of 100 patients who had brain trauma in young adult study in the Specialized Hospital Abel Gilbert Ponton period January to December 2015 were studied. Results: Adequate control of risk factors diminish index of head trauma in adult and its complications.

Keywords: Trauma, Brain Injury, Factors of Risk, Complications, Adult, Young Man.

\section{RESUMO}

Introdução: A lesão cerebral traumática é um problema mais relevante em todo o mundo que afeta principalmente a população masculina jovem, nosso estudo é orientado à análise de fatores de risco e complicações do TCE, em nossa incidência por tipo de lesão o TEC é considerável, o que pode elevar a taxa de morbimortalidade se não for diagnosticada e tratada precocemente. Metodologia: Estudo observacional transversal, retrospectivo, onde são estudados fatores de risco e complicações de traumatismo craniano em adultos. Foram estudados 100 pacientes que sofreram trauma cerebral no estudo de jovens adultos no período de janeiro a dezembro de 2015 no Hospital Especializado Abel Gilbert Ponton. Resultados: O controle adequado dos fatores de risco diminui o índice de traumatismo craniano no adulto e suas complicações.

Palavras-chave: Trauma, Lesão Cerebral, Fatores de Risco, Complicações, Adulto, Jovem. 


\section{Introducción}

El traumatismo craneoencefálico ha cobrado la vida de la mayor parte de la población adulto joven, constituyéndose la primera causa de muerte y discapacidad en personas menores de 46 años en el mundo entero, representando un grave problema de salud pública. (Mosquera Betancourt \& Vega Basulto, 2009) (Bárcena-Orbe, Rodríguez-Arias, Rivero-Martín, Cañizal-García, \& Mestre-Moreiro, 2006)

El traumatismo craneoencefálico es un deterioro funcional del contenido craneal causado por un intercambio brusco de energía mecánica, provocando conmoción contusión, hemorragia o laceración del cerebro. La severidad del trauma dependerá del tipo de la lesión. En países desarrollados o se encuentra un porcentaje del 40 al $60 \%$ de los casos, y el país en vías de desarrollo se encuentra una incidencia del $80 \%$ se puede determinar la gravedad de la lesión por medio de la medición de los niveles de consciencia utilizando la Escala de como de Glasgow dividiéndose en grave moderado y leve. (Valarezo Chuchuca, 2004) (Wegner \& Wilhelm, 2003)

Entre los factores de riesgo que están presentes en el traumatismo craneoencefálico la edad, presencia de lesión cerebral previa gravedad de la lesión, duración del coma, de la lesión duración de ingreso, epilepsia, shock hipovolémico (Cutillas, Traumatismo craneoencefalico, 2013). Entre las causas más comunes del traumatismo craneoencefálico en un individuo en adulto-joven los principales son: Accidentes de tráfico con un porcentaje del $75 \%$, caídas con un porcentaje del $20 \%$, lesiones deportivas con el $5 \%$, manifestándose lesiones primarias o secundarais. (Sallán Pueyo, 2019)

Aunque las complicaciones son poco comunes, el riesgo aumenta según la gravedad del trauma. Las complicaciones del traumatismo cerebral incluyen convulsiones inmediatas, hidrocefalia o engrandecimiento ventricular post-traumático, derrames de fluido cerebro espinal, infecciones, lesiones vasculares, lesiones del nervio craneal, dolor, úlceras por presión, disfunción, falla orgánica múltiple en pacientes inconscientes, y politrauma (Bethesda, 2010) (Andrés Matos, Vázquez Torres, Verdecia Sánchez, \& Zaldívar Santiesteban, 2011)

El propósito de este trabajo investigativo es determinar la influencia de los factores de riesgo en el desarrollo de las complicaciones del traumatismo cráneo encefálico en adulto-joven en nuestra población, para así establecer medidas que disminuyan la prevalencia de este tipo de traumatismo a nivel local.

\section{Metodología}

\section{Tipo de investigación}

El siguiente trabajo es de tipo descriptivo retrospectivo.

\section{Diseño de Investigación}

No experimental, de cohorte transversal

Procedimientos

Consideraciones éticas: Primeramente, entregamos un oficio con el tema del proyecto al director del Hospital Especializado Abel Gilbert Pontón para la autorización. Y se elaborara un consentimiento para garantizar confidencialidad de los pacientes que presentaron los factores de riesgo y complicaciones del traumatismo craneoencefálico sobre de los datos obtenidos

\section{Procedimientos de datos}

Una vez conseguida la información de la muestra se puntualizará el cruce de las variables dependientes e independientes y los criterios para constituir los datos obtenidos en el trabajo de campo teniendo como referencia los indicadores, utilizando el programa de cómputo Excel y los resultados serán expuestos en las tablas y gráficos.

\section{Técnicas para obtener los datos}

Esta técnica se realizará mediante recolec- 
ción de historiales médicos y una observación directa, esta técnica será la de mayor uso que nos permitirá estar en contacto con los hechos y acontecimientos para captar información muy valiosa para la ejecución del proyecto y el informe final.

\section{Resultados}

\section{Traumatismo craneoencefálico - distri- bución por edad en adulto joven}

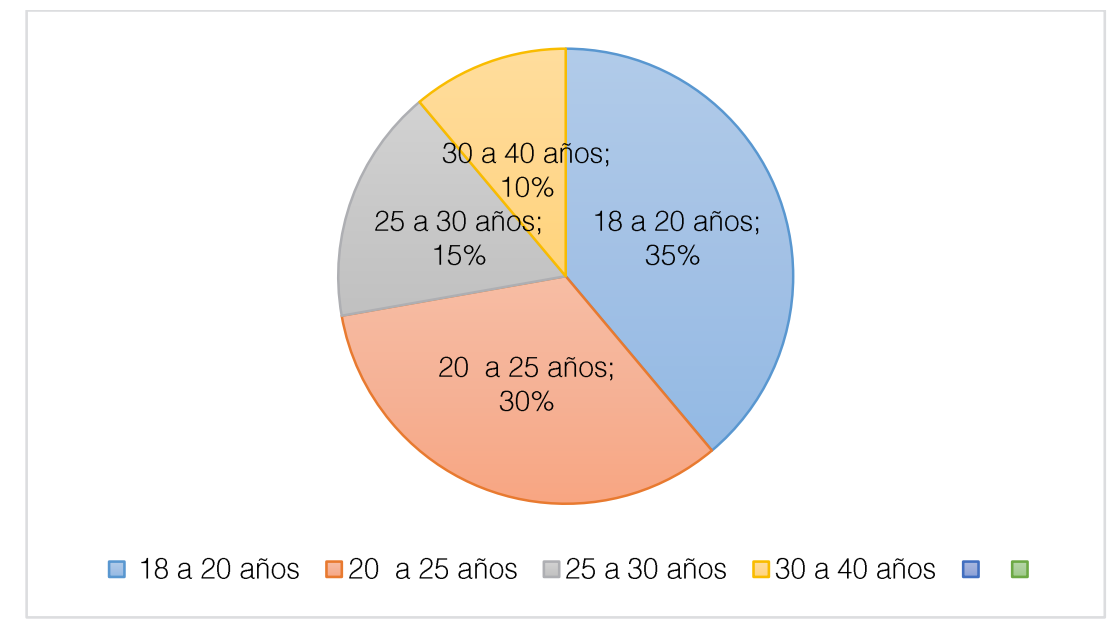

\section{Gráfico 1. Edad}

Fuente: Datos estadísticos del Hospital Especializado Abel Gilbert Pontón

Análisis: El resultado de análisis revela el índice de incidencia del TRAUMATISMO CRANEOENCEFÁLICO por edad del adulto mayor, en primer lugar lo ocupa el grupo comprendido de 18 a 20 (35\%), seguido 20 a 25 (30\%), en cuarto lugar lo ocupa el gru- po de edades de 30 a 40 años de edad que representa el grupo de adultos jóvenes que fueron atendidos con TRAUMATISMO CRANEOENCEFÁLICO por factores de riesgo y complicaciones presente.

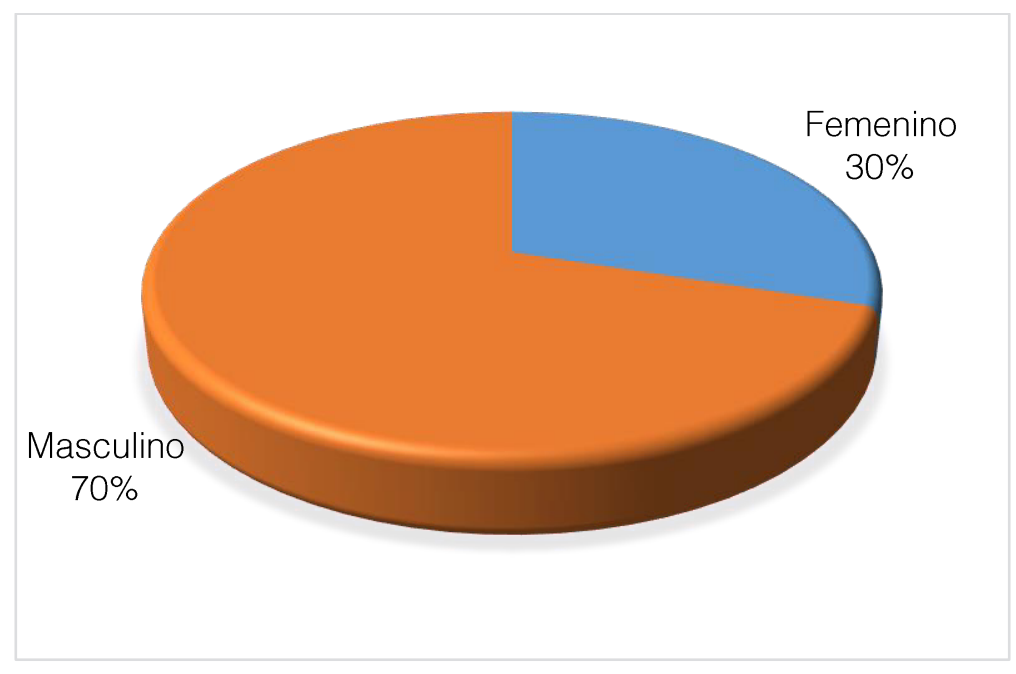

Gráfico 2. Traumatismo craneoencefálico - distribución por sexo

Fuente: Datos estadísticos del Hospital Especializado Abel Gilbert Pontón 
Análisis: El análisis de resultado revela que entre existe mayor incidencia de trauma cráneo encefálico en varones (70\%), siendo la población con mayor riesgo de presentar este tipo de trauma debido a los factores de riesgo presentes en el medio, acciden- tes automovilísticos, accidentes laborales, o accidentes dentro del hogar, entre otros, en diferencia con el sexo femenino (30\%), ellas representa a la población con menos riesgo.

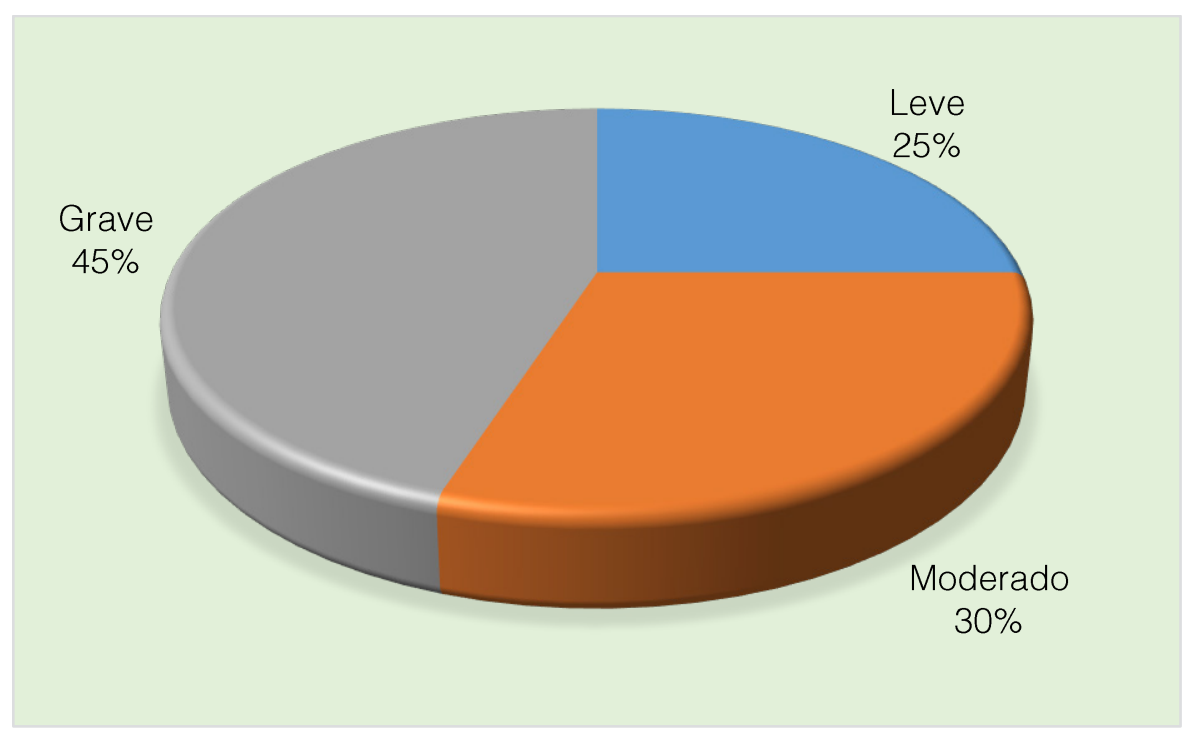

Gráfico 3. Distribución por tipo de traumatismo craneoencefálico

Fuente: Datos estadísticos del Hospital Especializado Abel Gilbert Pontón

Análisis: El análisis de resultado que la mayor prevalencia de recién nacidos y niños que contrajeron este síndrome de inmunodeficiencia por vía vértical predomina el sexo masculino con un $55 \%$, seguido del sexo femenino con un $45 \%$, los cuales de- ben someterse a un tratamiento continuo de esta enfermedad para evitar complicaciones futuras y disminuir el índice de mortalidad infantil por este síndrome.

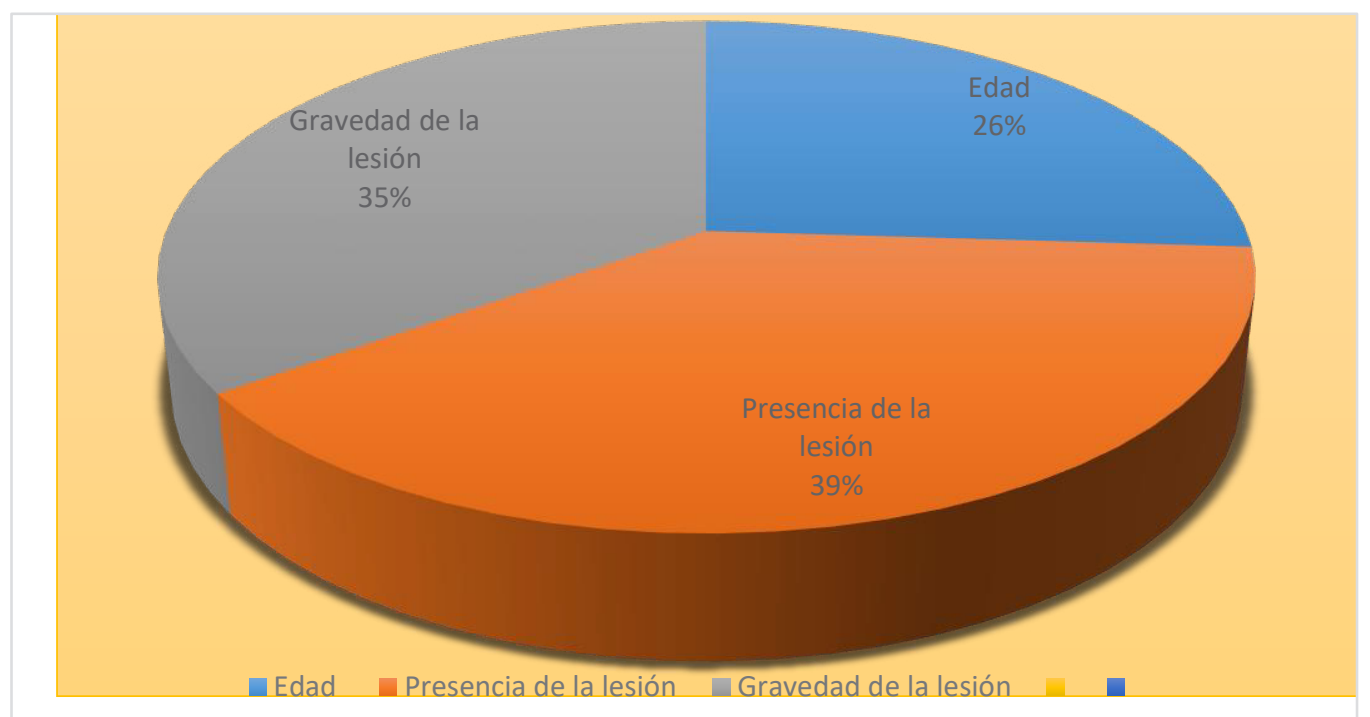

Gráfico 4. Traumatismo craneoencefálico - distribución por factores de riesgo

Fuente: Datos estadísticos del Hospital Especializado Abel Gilbert Pontón 
Análisis: El análisis de resultado muestra los principales factores de riesgo presentes que se encierro en tres principales: por edad (26\%) debido a que la edad constituye uno de los factores principales al TRAUMATISMO CRANEOENCEFÁLICO, presencia de la lesión (39\%) el tipo de lesión juega un papel fundamental, pues el individuo puede estar propenso a los accidentes automovilísticos, laborales o dentro del hogar lo que puede llevar a estos tipos de trauma, la gravedad de la lesión (35\%) según la escala de Glasgow constituye un factor de riesgo en personas que presentan TRAUMATISMO CRANEOENCEFÁLICO.

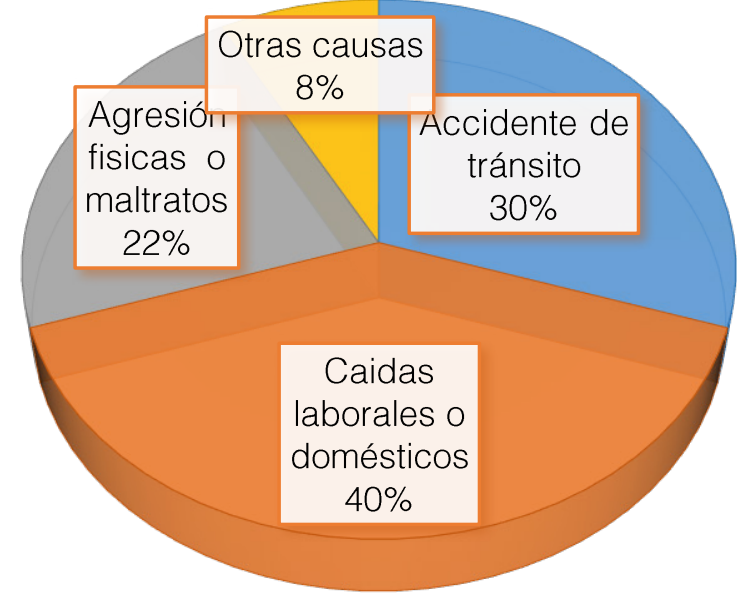

Gráfico 5. Mecanismos causantes del traumatismo craneoencefálico

Fuente: Datos estadísticos del Hospital Especializado Abel Gilbert Pontón

Análisis: El análisis de resultado revelo que los mecanismos causantes del TRAUMATISMO CRANEOENCEFÁLICO tienen gran incidencia en nuestro medio, como por ejemplo, las caídas laborales o domésticas (40\%) siguen ocupando el primer lugar en la presencia de TRAUMATISMO CRANEOENCEFÁLICO, en segundo lugar lo ocupa los accidentes de tránsitos (30\%) que en países desarrollado es la causa número uno de TRAUMATISMO CRANEOENCEFÁLICO y de muertes, seguido por la agresión a maltrato físico (22\%) en un medio donde existe la violencia familiar y social, y entre otros que encierra los juegos bruscos, caídas leves accidentes con objetos o animales.

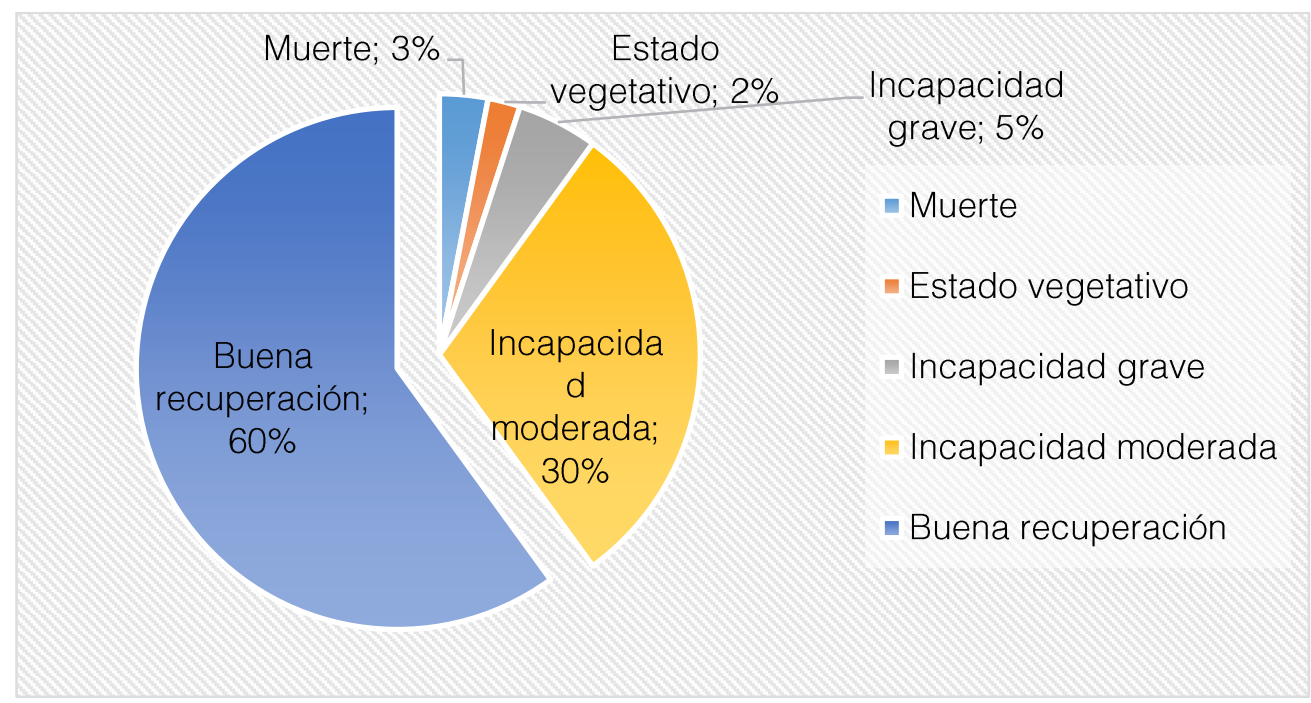

Gráfico 6. Traumatismo craneoencefálico - escala de severidad

Fuente: Datos estadísticos del Hospital Especializado Abel Gilbert Pontón 
Análisis: El análisis de resultado revelo el grado de severidad de traumatismo cráneo encefálico con mayor prevalencia en nuestro medio, un pequeño porcentaje revelo que no existe un número significativo de estado vegetativo, seguido de muertes por TRAUMATISMO CRANEOENCEFÁLICO (3\%), en tercer lugar por tipo se severidad , la incapacidad grave (5\%), mientras que la incapacidad leve o moderada ocupa el segundo lugar de incidencias (30\%), y con mayor frecuencia esta la buena recuperación del paciente (60\%) debido al manejo y control oportuno de los factores de riesgo, y de médicos profesionales en el área, se reduce en gran medida en índice de morbilidad y mortalidad ven individuos adultos-joven.

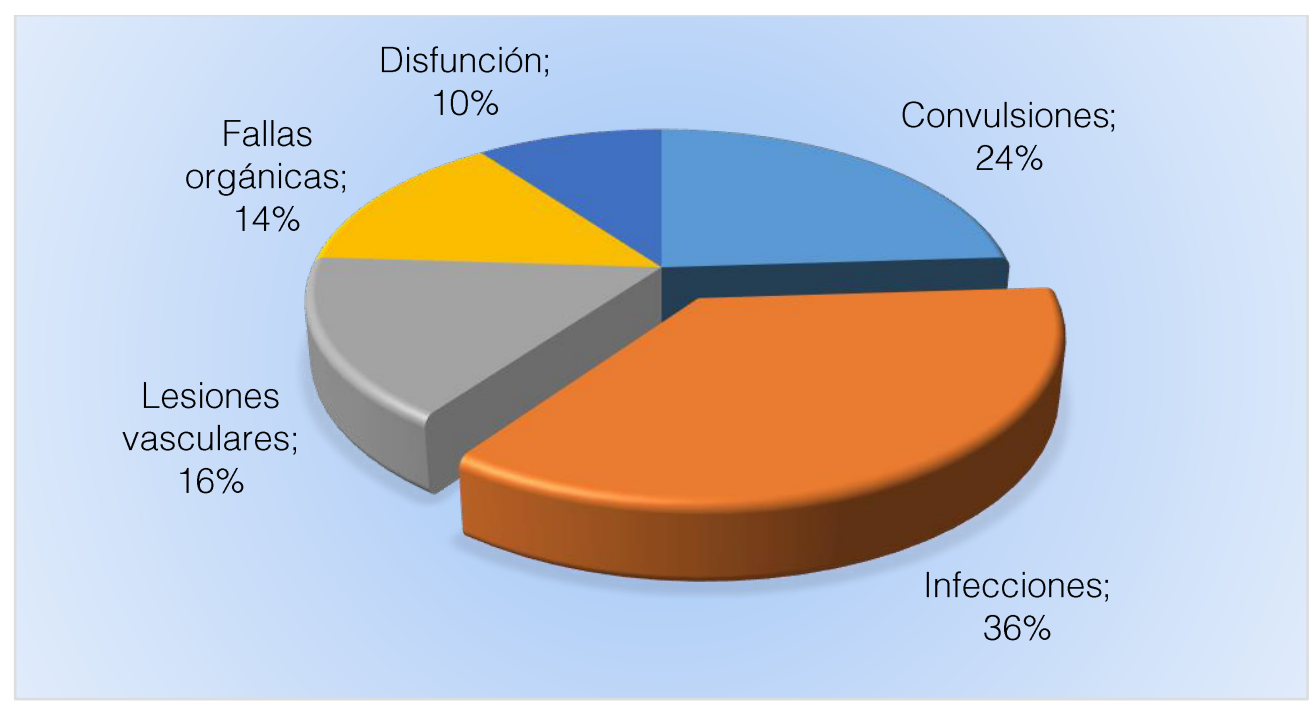

Gráfico 7. Traumatismo craneoencefálico - escala de complicaciones

Fuente: Datos estadísticos del Hospital Especializado Abel Gilbert Pontón

Análisis: El análisis de resultado revelo que entre las complicaciones que se manifiestan en el TRAUMATISMO CRANEOENCEFÁLICO están: infecciones (36\%), convulsiones
(24\%), lesiones vasculares (16\%), fallas orgánicas (14\%), y el índice de disfunciones se redujo (10\%) considerablemente.

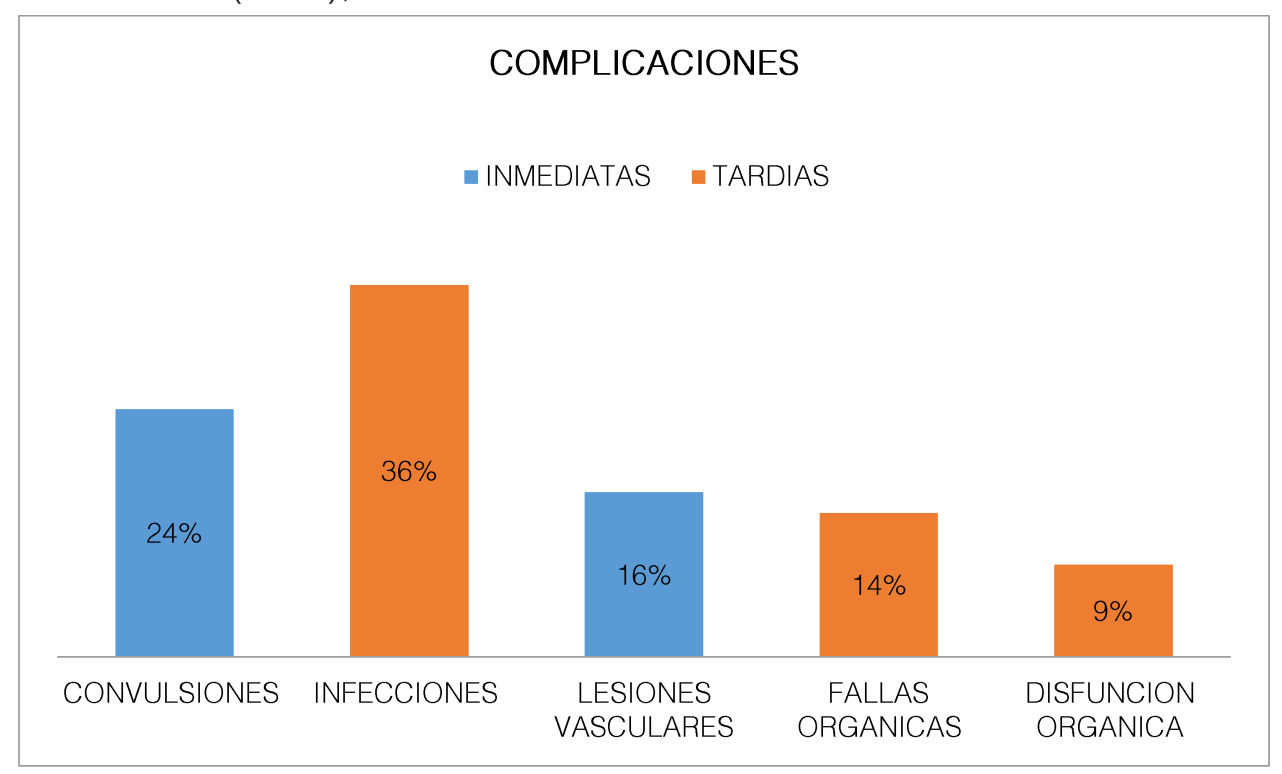

Gráfico 8. Complicaciones

Fuente: Datos estadísticos del Hospital Especializado Abel Gilbert Pontón 
Análisis: El análisis de resultado revelo que entre las complicaciones inmediatas fueron las convulsiones con $24 \%$, lesiones vasculares con 16\%, considerablemente. Las complicaciones tardías fueron infecciones $36 \%$, fallas organicas $14 \%$, disfunción organica $9 \%$.

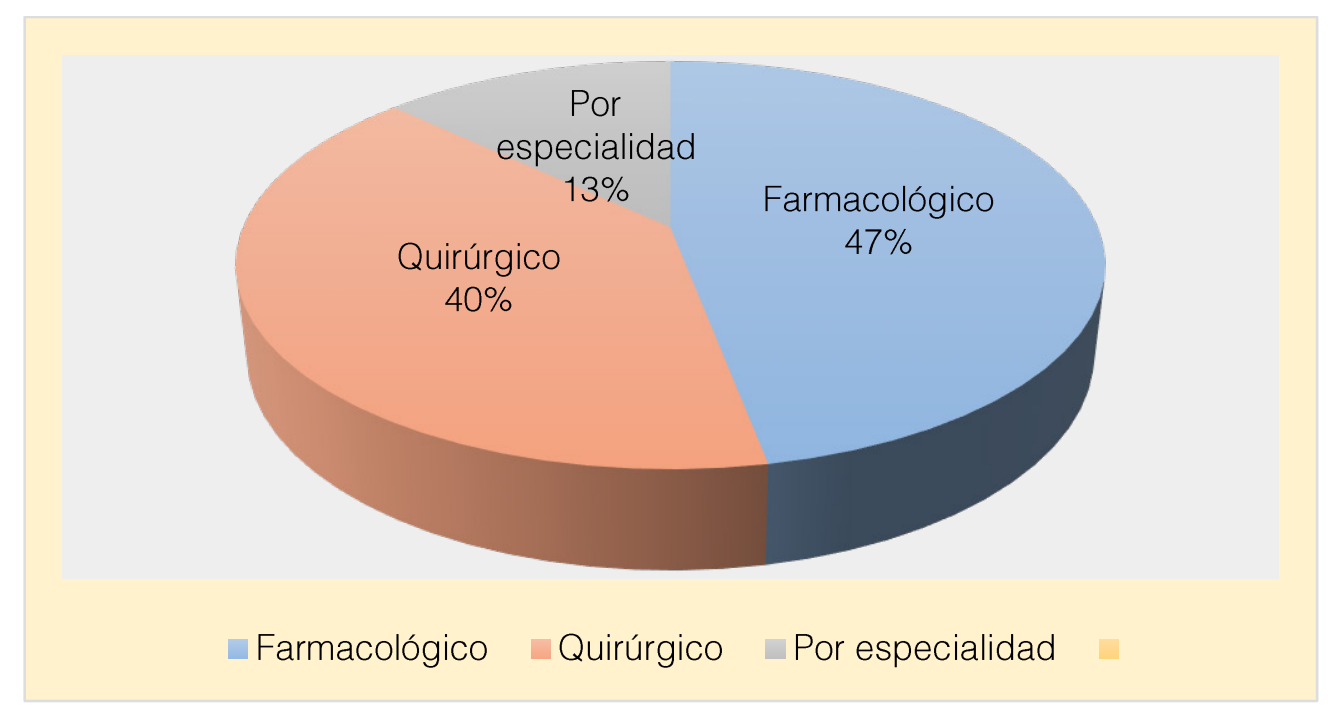

Gráfico 9. Tratamiento oportuno a los tipos de traumatismo craneoencefálico

Fuente: Datos estadísticos del Hospital Especializado Abel Gilbert Pontón

Análisis: El análisis de resultado revelo que los tratamientos oportunos a los tipos de TRAUMATISMO CRANEOENCEFÁLICO mas aplicado es el farmacológico (47\%), por ser de tipos leves y moderados, seguido por el método quirúrgico (40\%) cuan- do se manifiesta el tipo de TRAUMATISMO CRANEOENCEFÁLICO grave, y por especialidad (13\%) en caso de que se presente el TRAUMATISMO CRANEOENCEFÁLICO de cualquier topo y amerite oros tipos de tratamiento.

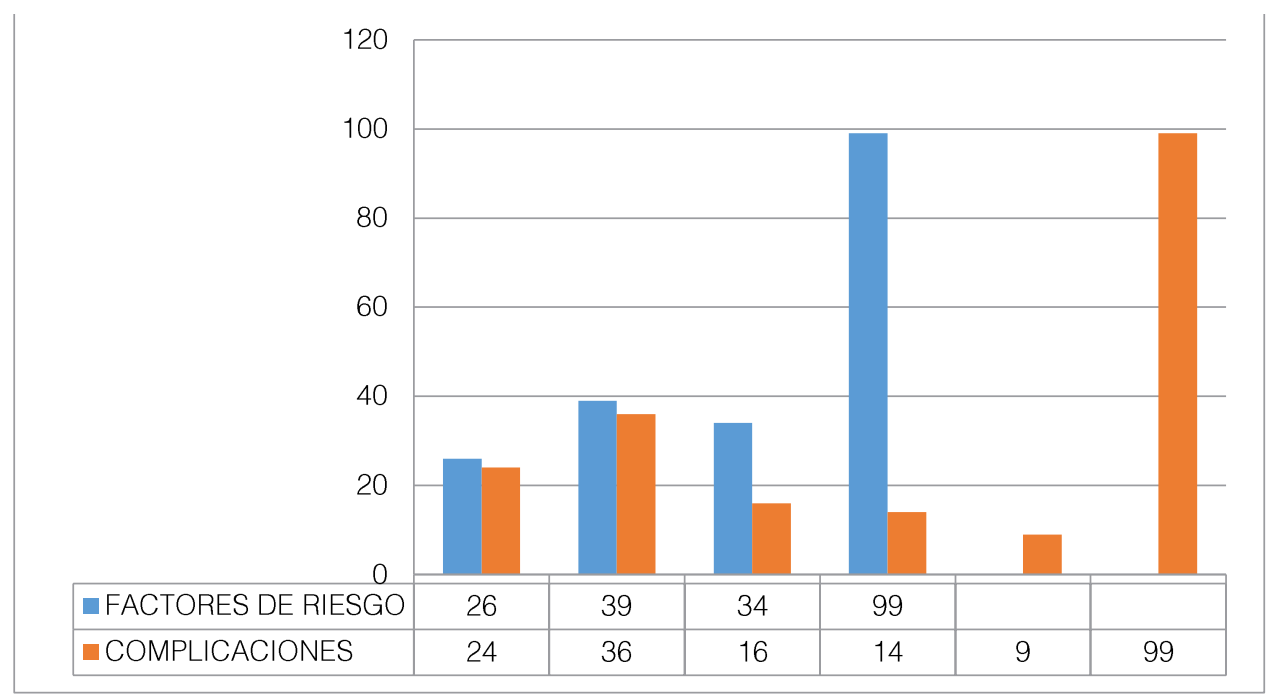

Gráfico 10. Tratamiento oportuno a los tipos de traumatismo craneoencefálico

Fuente: Datos estadísticos del Hospital Especializado Abel Gilbert Pontón 
Análisis: El análisis de resultado revelo existe una correlación entre los factores de riesgo y las complicaciones, después de hacer el análisis de las tablas estudiadas anteriormente.

\section{Conclusiones}

Con este trabajo de investigación realizado se llegó a la conclusión del que el trauma cráneo encefálico constituye un problema de mayor relevancia en el mundo entero, debido a la severidad deja secuelas físicas y mentales en la persona que presenta este tipo de trauma

En nuestra investigación encontramos que el sexo masculino tiene mayor incidencia con un $70 \%$, mientras que el femenino $29 \%$, la edad más afectada fue de 20-25 años, con una media de 22 años con el $30 \%$ de todos los casos.

En nuestra investigación encontramos que las principales mecanismos causantes del trauma craneoencefálico fueron: accidente de tránsito 30\%, caídas laborales o domesticas $40 \%$, agresión física $22 \%$, otras causas $8 \%$.

Las complicaciones fueron convulsiones $24 \%$, infecciones $36 \%$, lesiones vasculares $16 \%$, fallas orgánicas $14 \%$, disfunción orgánica $10 \%$ las cuales se dividen en inmediatas y tardías.

La incidencia va en aumento en relación con los años anteriores, de acuerdo al número de casos registrados.

Se logró encontrar una correlación entre los factores de riesgo y las complicaciones, en el cual las complicaciones aumentaban de acuerdo al mecanismo de la lesión y al tiempo de traslado del paciente.

Se desea llegar a la población más vulnerable e informar sobre los tipos de lesiones del trauma cráneo encefálico y brindarles la información oportuna sobre el tema pata que sepan reconocer los posibles factores de riesgo y las complicaciones que se ma- nifiesta, con el fin de reducir el índice de morbilidad y mortalidad asociada a este evento de todos los pacientes que llegan al área de emergencia del hospital Abel Gilbert Pontón.

\section{Bibliografía}

Andrés Matos, A., Vázquez Torres, C., Verdecia Sánchez, L., \& Zaldívar Santiesteban, M. (2011). Complicaciones del trauma craneoencefálico severo en la unidad de cuidados intensivos pediátricos. Revista Cubana de Pediatría, 83(3), 236-247. Obtenido de http://scielo.sld.cu/scielo.php?script=sci_arttext\&pid=S0034-75312011000300003

Bárcena-Orbe, A., Rodríguez-Arias, C., Rivero-Martín, B., Cañizal-García, J., \& Mestre-Moreiro, C. (2006). Revisión del traumatismo craneoencefálico. Neurocirugía, 17(6), 495-518. Obtenido de http://scielo.isciii.es/scielo.php?script=sci_arttext\&pid=S1130-14732006000600001

Bethesda, M. (Junio de 2010). ¿Qué complicaciones inmediatas posteriores al trauma pueden ocurrir con traumatismo cerebral? Obtenido de brainline.org: http://www.brainline.org/content/2010/06/qu-complicaciones-inmediatas-posteriores-al-trauma-pueden-ocurrir-con-traumatismo-cerebral_pageall.html

Cutillas, M. M. (2013). Traumatismo craneoencefalico. Master Neuropsicología Clínica, 5.

Mosquera Betancourt, G., \& Vega Basulto, S. (2009). Mortalidad por trauma craneoencefálico en el adulto mayor. Revista Archivo Médico de Camagüey, 13(1). Obtenido de http:// scielo.sld.cu/scielo. php?script=sci_arttext\&pi$d=S 1025-02552009000100007$

Sallán Pueyo, A. (2019). Intervención para el manejo de un traumatismo craneoencefálico en el servicio de emergencias prehospitalarias. Lérida, España: Universitat de Lleida. Obtenido de https://repositori.udl.cat/bitstream/handle/10459.1/66651/asallanp. pdf?sequence $=1$ \&isAllowed $=y$

Valarezo Chuchuca, A. (2004). El traumatismo craneoencefálico. Guayaquil: Médicos Ecuador. Obtenido de medicosecuador.com: http://www. medicosecuador.com/espanol/articulos/accidentes-cabeza.html

Wegner, A., \& Wilhelm, J. (2003). Traumatismo encefalocraneano: Conceptos fisiológicos. Revista chilena de pediatría, 74(1), 16-30. Obtenido de https://scielo.conicyt.cl/scielo.php?script=sci_arttext\&pid=S0370-41062003000100003 


\section{CITAR ESTE ARTICULO:}

Paredes Zambrano, K., Cedeño Veintimilla, M., De Los Ríos Tomalá, P., \& Vaca Morla, F. (2020). Factores de riesgo y complicaciones del traumatismo craneoencefálico en adulto joven. RECIMUNDO, 4(1), 142-151. doi:10.26820/ recimundo/4.(1).enero.2020.142-151

\section{(c) $(\oplus \otimes \odot$ \\ BY NC SA}

RECONOCIMIENTO-NOCOMERCIAL-COMPARTIRIGUAL CC BY-NC-SA

ESTA LICENCIA PERMITE A OTROS ENTREMEZCLAR, AUUSTAR Y CONSTRUIR A PARTIR DE SU OBRA CON FINES NO COMERCIALES, SIEMPRE

Y CUANDO LE RECONOZCAN LA AUTORÍA Y SUS NUEVAS CREACIONES ESTÉN BAJO UNA LICENCIA CON LOS MISMOS TÉRMINOS. 